\section{Chávez-Lazo YE 1 \\ Rodríguez-Cárdenas YA ${ }^{2}$ Ruíz-Mora GA ${ }^{3}$}

1 Maestría en Radiología Bucal y Maxilofacial, Escuela de Odontología, Universidad Científica del Sur - UCSUR, Lima, Perú

2 Profesor Asociado de la Maestría en Radiología Bucal y MaxiloFacial de la Escuela de Odontología, Universidad Científica del Sur - UCSUR, Lima, Perú.

3 Profesor Asociado de la Maestría en Radiología Bucal y MaxiloFacial de la Escuela de Odontología, Universidad Científica del Sur - UCSUR, Lima, Perú y Universidad del Bosque, Bogotá, Colombia.

\title{
ATEROMAS COMO HALLAZGO INCIDENTAL EN TOMOGRAFÍA COMPUTARIZADA DE HAZ CÓNICO (TCHC): REPORTE DE UN CASO
}

\section{RESUMEN}

Los ateromas son lesiones focales que se desarrollan cuando las sustancias grasas, plaquetas, residuos celulares, y el calcio se depositan en el revestimiento de las arterias, siendo la causa principal de los infartos de miocardio y accidentes cerebrovasculares. Estas enfermedades implican una secuencia de factores biológicos y mecanismos que pueden estar influenciados por diabetes mellitus, obesidad, hipertensión arterial, y una inadecuada dieta. Se reporta el caso de una paciente de 76 años, con imágenes nodulares hiperdensas bilaterales bien definidas sobre la TCHC, la cual permitió observar la localización exacta de las calcificaciones en los espacios de la orofaringe y carótida. Se llegó a un diagnóstico radiológico presuntivo de ateromas calcificados de carótidas. Este hallazgo incidental de calcificaciones bilaterales podría ser el indicador de una situación adversa en el futuro del paciente, ya que a menudo son manifestaciones de enfermedades silenciosas que pueden conducir a la muerte.

PALABRAS CLAVE: Ateroma Carotídeo, Factores de Riesgo, Aterosclerosis Carotídea, Tomografía Computarizada de Haz Cónico.

\section{ATHEROMAS AS INCIDENTAL FINDINGS IN CONE BEAM COMPUTERIZED TOMOGRAPHY: A CASE REPORT}

\section{Abstract}

The atheromas are focal lesions that develop when fats, platelets, cellular debris and calcium substances are deposited on the lining of the arteries, being the cause principal of heart attacks and strokes. This diseases involves a sequence of biological factors and mechanisms which are influenced by diabetes mellitus, obesity, hypertension, poor diet and eating habits which are the classic risk factors that aggravate the disease. The case of a patient of 76 years is reported, with well-defined bilateral hyperdense nodular lesions on the CBCT, which allowed us to observe the exact location of calcifications in the spaces of the oropharynx and carotid. It was a presumptive radiological diagnosis of calcified carotid atheroma. This incidental finding of calcifications bilateral determines a powerful indicator of an adverse situation in the future of the patient, as they often are indicators of diseases quiet that can lead to death. 


\section{INTRODUCCIÓN}

Los ateromas son lesiones focales (características de la aterosclerosis) que se desarrollan cuando las sustancias grasas, plaquetas, residuos celulares, y el calcio se depositan en el revestimiento de las arterias, aumentando su grosor en su parte interna. Otros factores como el tabaquismo y la menopausia pueden conducir al desarrollo de daño endotelial y aterogénesis..$^{1,2,3}$ Específicamente los ateromas de carótida se han asociado fuertemente con la enfermedad arterial coronaria, y con accidentes cerebro-vasculares e infarto cerebral, causando una embolia en la bifurcación de la arteria carótida común, o de la porción proximal de la arteria carótida interna ocluyendo una rama de la arteria cerebral media. ${ }^{4,5}$

Friedlander y et al. ${ }^{6-8}$ fueron los primeros en describir la presencia de calcificaciones en el área de la arteria carótida mediante radiografías panorámicas realizadas para el diagnóstico dental de rutina, y encontraron una prevalencia de aproximadamente $2 \%$ en una muestra de 1000 pacientes de edad avanzada. La detección de estas lesiones en las radiografías panorámicas en la población dental general también oscila entre el $3 \%$ y $5 \% .^{10,11-14}$
Numerosos estudios reportan la localización más frecuente como la zona postero-inferior del ángulo de la mandíbula, aproximadamente a nivel del margen inferior de la tercera vértebra cervical (entre C3 y C4), o a nivel o por debajo del hueso hioides. ${ }^{9,15-17}$ Las placas de ateromas aparecen en las imágenes de las radiografías panorámicas como masas nodulares heterogéneas y radiopacas o como líneas verticales también radiopacas, por la calcificación de las mismas, siguiendo el trayecto del vaso sanguíneo que posteriormente son diagnosticadas como ateromas mediante Color Doppler, que vendría a convertirse en el gold standar. ${ }^{17}$ Para el análisis y el diagnóstico diferencial de las imágenes con radiografías panorámicas, es importante tener en cuenta una serie de estructuras anatómicas en la región del cuello, tales como: el hueso hioides, apófisis estiloides, cartílago tritíceo, cartílago tiroides, la epiglotis, los ligamentos estilo-hioides y estilomandibular. $18,19,20,21$

Es importante tener en cuenta otras hallazgos patológicos que se pueden presentar en la misma localización sobre una radiografía panorámica, y que pueden ocasionar confusión en el diagnóstico, tales como: (Tabla 1)

Tabla 1 Calcificaciones Distróficas ${ }^{22,23}$

\begin{tabular}{|l|l|}
\hline $\begin{array}{l}\text { Nodos Linfáticos } \\
\text { calcificados }\end{array}$ & $\begin{array}{l}\text { En la radiografía panorámica se evidencian como masas } \\
\text { radiopacas heterogéneas e irregulares, unilaterales o } \\
\text { bilaterales, llamadas también masas en coliflor, las cuales se } \\
\text { observan proyectadas sobre la rama y ángulo mandibular, o por } \\
\text { debajo de los mismos. }\end{array}$ \\
\hline Tonsilolitos & $\begin{array}{l}\text { En radiografías panorámicas generalmente se observan } \\
\text { formaciones pequeñas y múltiples sobre proyectadas en la parte } \\
\text { media de la rama mandibular, de contornos mal definidos y de } \\
\text { densidad ligeramente más radiopaca que el hueso esponjoso. }\end{array}$ \\
\hline
\end{tabular}

Calcificaciones Idiopáticas

\begin{tabular}{|l|l|}
\hline Flebolitos & $\begin{array}{l}\text { Estas calcificaciones pueden verse en radiografías panorámicas } \\
\text { y postero-anteriores, presentándose como múltiples cuerpos } \\
\text { laminados de forma circular u ovalada con un rasgo } \\
\text { característico de halo radiopaco en la periferia y radiolúcido en } \\
\text { centro, con aspecto radiopaco homogéneo interior y apariencia } \\
\text { de láminas concéntricas. }\end{array}$
\end{tabular}


Los ateromas son calcificaciones que no siempre pueden ser identificadas con una radiografía panorámica, sobre todo en cuanto a su ubicación exacta y al grado de oclusión. ${ }^{9}$ Puede confundirse con otras imágenes o simplemente no visualizarse nítidamente. Este aspecto es superado ampliamente con el uso de una Tomografía Computarizada de Haz cónico (TCHC). ${ }^{24,25} \mathrm{El}$ reconocimiento de la placa calcificada de la arteria carótida, puede ofrecer la oportunidad para la reducción de riesgo de accidente cerebrovascular. ${ }^{25-27}$ La TCHC está ganando un uso más generalizado en múltiples campos de la odontología y se constituye en una herramienta interesante y bienvenida. Sin embargo, hay escasa literatura sobre la anatomía tridimensional de estas estructuras y cómo se presentan cuando está calcificada.

En este reporte de caso de tipo radiológico se presenta una evaluación por TCHC de ateromas de la arteria carótida. El objetivo es brindar a los odontólogos generales y estudiantes, la identificación de sus características radiológicas en 3D, para de esta forma remitir al especialista, indicando la identificación de la imagen hiperdensa no compatible con los reparos anatómicos de la zona. El especialista se encargará de realizar pruebas más sensibles para confirmar o negar la sospecha del odontólogo.

\section{REPORTE DEL CASO}

Paciente femenino de 76 años de edad, natural del departamento de Lima, que acudió al centro radiológico de imágenes maxilofaciales referido por el especialista, para evaluación diagnóstica de implantes en el maxilar inferior.

Por protocolo, el Centro de Diagnóstico por Imágenes (CDI) del Dr. Hugo Aguayo, realiza una adquisición de imagen panorámica preliminar, sobre la cual se localizan como hallazgo incidental unas imágenes radiopacas de forma ovalada, compatibles con calcificaciones intravasculares de manera bilateral por debajo del ángulo mandibular y entre el hueso hioides, y la columna vertebral (espacio correspondiente entre C3 y C4) (Figura 1). A su vez, presenta opacificación bilateral de los senos maxilares, presencia de reabsorción de las crestas óseas alveolares, y restauraciones coronarias con posibles caries recidivantes en las piezas 15,25 , y 35 . En la pieza 24 se observa una reacción osteólitica a nivel apical. Las piezas 37 , 46 y 47 son portadoras de corona metal cerámica, así como la piezas 25 , y 45 portadoras de espigo muñón y corona metal cerámica.

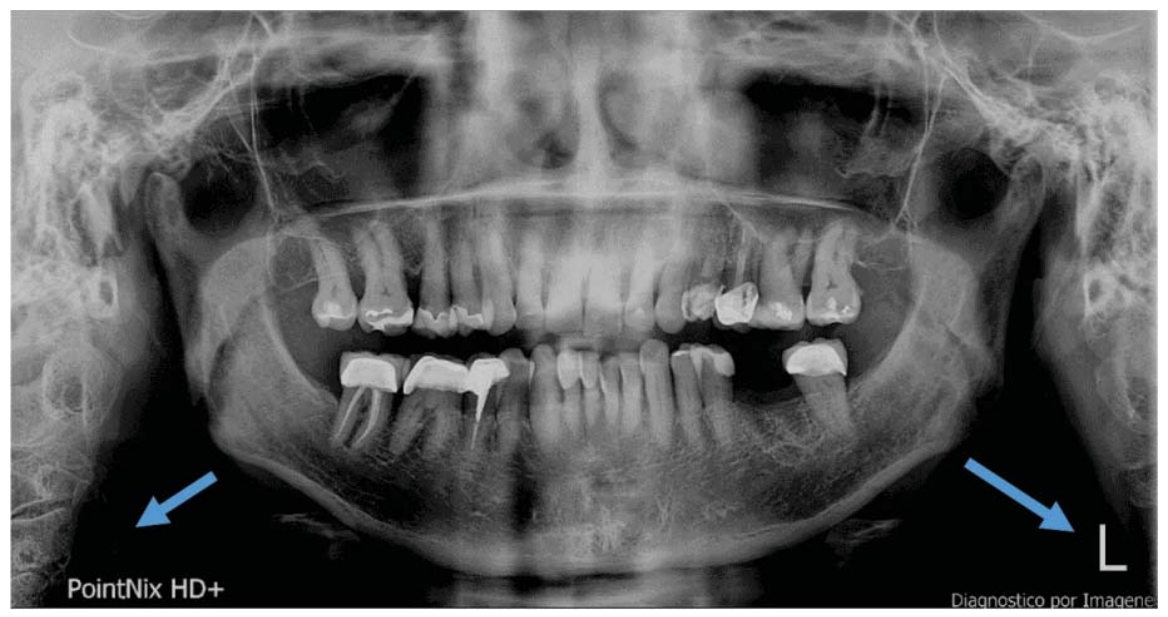

Figura 1.

En la panorámica observamos muy difusamente pequeñas calci caciones a la altura de la arteria carótida a la derecha e izquierda (Fechas azules). 


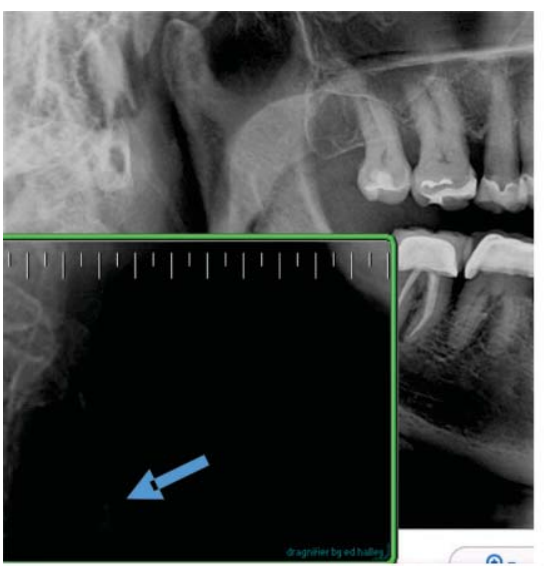

Figura: 1-A

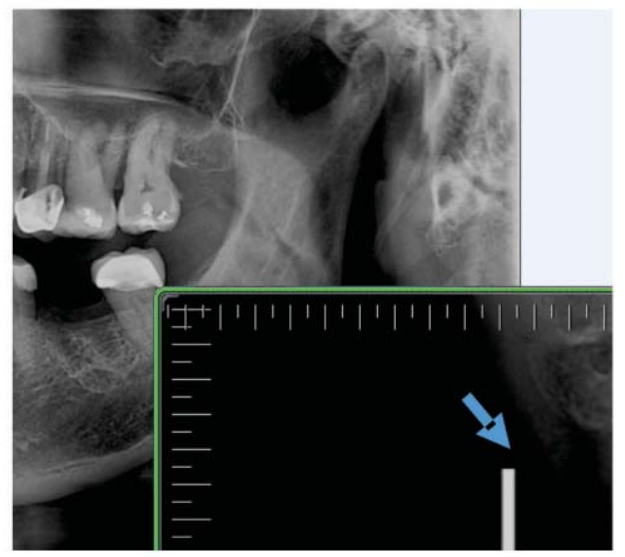

Figura: 1-B

En la imagen derecha se observa una radiopacidad de apariencia nodular con calcificaciones puntiformes de límites bien definidos y mucho más radiopaca que la del lado izquierdo (figura: 1-A). En el lado izquierdo se observa dos imágenes radiopacas de forma tubular bien delimitadas (figura: 1-B).

\section{Evaluación sobre la TCHC}

Las imágenes de TCHC fueron tomadas usando el Equipo Vatech E-Woo modelo Picasso Master, de origen coreano, operado a $90 \mathrm{kVp}$ y $8 \mathrm{~mA}$, con un tiempo de exposición de 20 segundos y un tamaño de voxel de $0.4 \mathrm{~mm}$. Todas las imágenes fueron tomadas siguiendo el protocolo recomendado. De acuerdo a los requerimientos, se usó un FOV de $20 \times 19 \mathrm{~cm}$. Las imágenes TCHC fueron analizadas con el software RealScan 2.0, mediante reconstrucción multiplanar en vistas axiales, coronales y sagitales, así como la evaluación mediante reconstrucciones $3 \mathrm{D}$ en volumen renderizado, en una estación de trabajo de Hp Intel CORE Ivpro, visualizadas en un monitor de 18 pulgadas con una resolución de 1366 x 768 pixels en un ambiente con luz tenue. El contraste y brillo de las imágenes fueron ajustados usando la herramienta procesadora de imágenes en el software para asegurar una visualización óptima.

La tomografia reveló en los diferentes cortes la presencia bilateral de una imagen hiperdensa de densidad calcifica, de aspecto homogeneo de forma nodular que se identifica con flechas celestes, corte axial (Figura 2), corte coronal (Figura 3), en las imágenes de maxima intensidad sagitales (Figuras 4 y 5) y en las recontruciones tridimencionales (Figura 6).

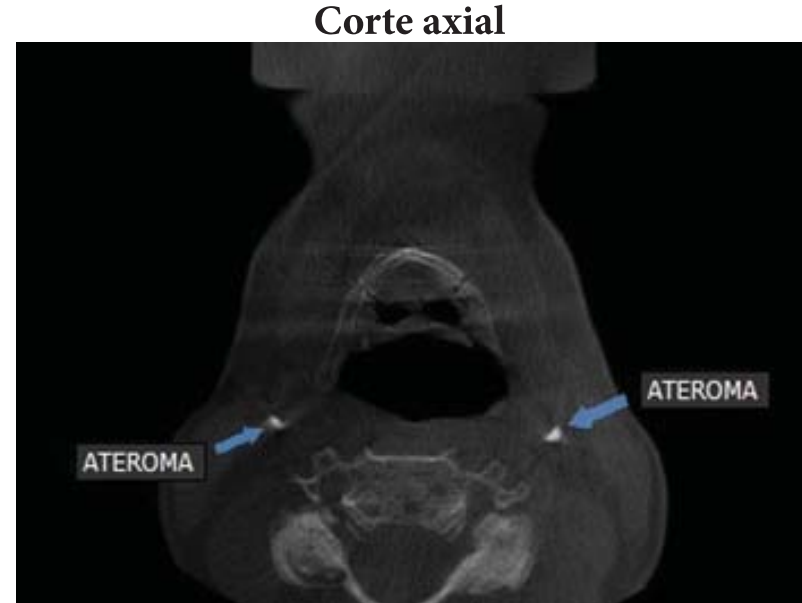

Figura 2. Se observa con mayor hiperdensidad la presencia de calcificaciones en ambos lados (derecho e izquierdo) bien circunscritos.

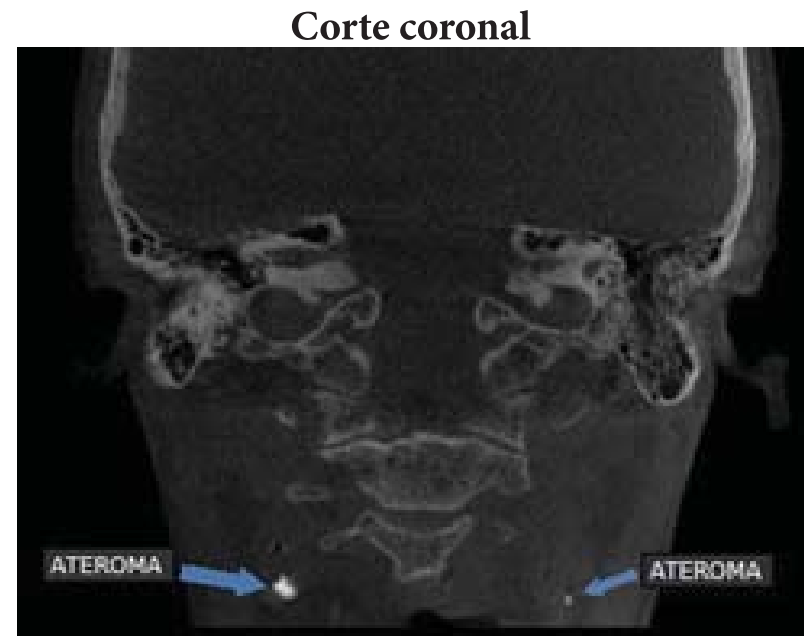

Figura 3. En este corte se observa la calcificación en el lado derecho mucho más grande e hiperdenso que en el lado izquierdo que es mucho más pequeño. 


\section{Maxima intensidad sagital}

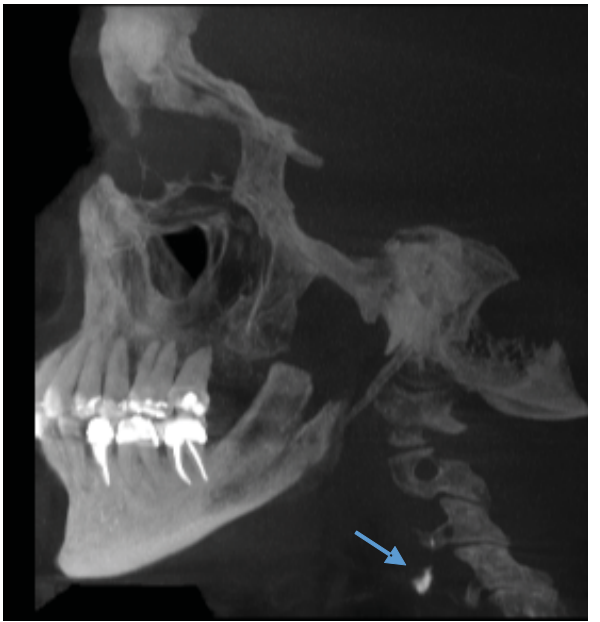

Figura 4. Se observa una masa hiperdensa bien circunscrita (Flecha celeste)

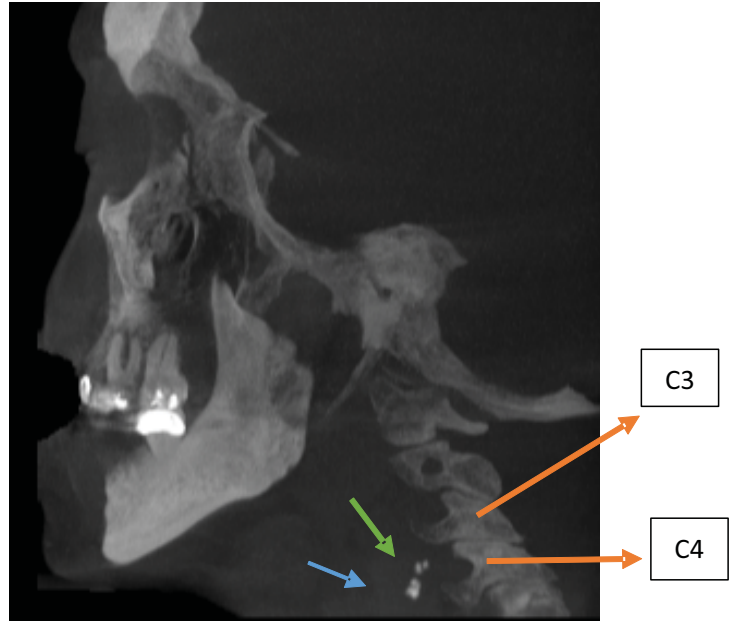

Figura 5. Se observa varias calcificaciones una grande (flecha celeste), que otras con forma de líneas verticales mucho más pequeñas (flecha verde), a la altura de C3 y C4 (flechas naranjas).

\section{Volumen Renderizado o reconstrucción tridimensional.}

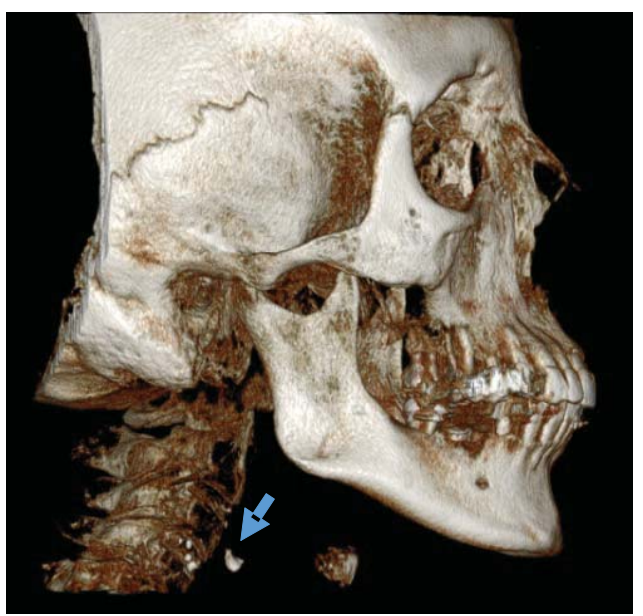

Figura 6. Se observa claramente las calcificaciones por debajo del ángulo mandibular a la altura de la $\mathrm{C} 3$ y $\mathrm{C} 4$.

El diagnóstico radiológico diferencial de las calcificaciones presentadas bilateralmente en TCHC fue de ateromas, ya que su localizaban en la región carotidea descarta las otras calcificaciones de tejido blando que se presentan a nivel maxilofacial.

\section{DISCUSIÓN}

La aterosclerosis es la causa principal de los in-

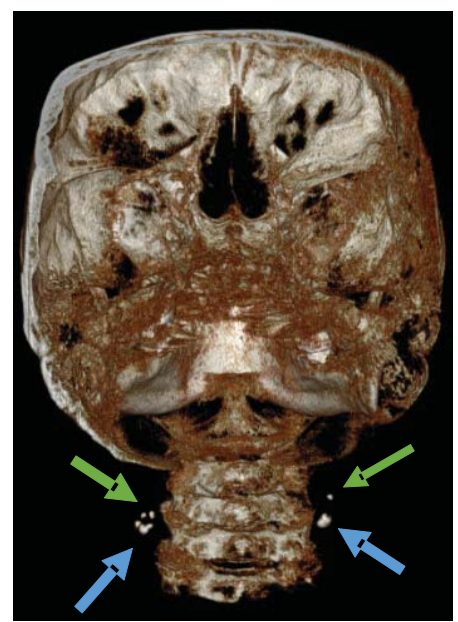

Figura 7. Se observa los nódulos calcificados uno de mayor tamaño (flecha celeste) y otros más pequeños (flecha verde).

fartos de miocardio y accidentes cerebrovasculares en la población mundial, y determinan la muerte de miles de personas. La enfermedad presenta una compleja patogénesis que implica una secuencia de factores biológicos y mecanismos que están influenciados por la diabetes mellitus, obesidad, hipertensión arterial, una inadecuada dieta y hábitos alimentarios, los cuales que son factores de riesgo clásicos que agravan esta enfermedad. Por medio de nuevas investigaciones, se 
han reportado otros factores desfavorables para su desarrollo, tales como enfermedad renal crónica, menopausia, y la apnea obstructiva del sueño (apnea del sueño). ${ }^{21,24,28,29}$

La evaluación de este caso radiográfico muestra la forma como se observan los ateromas calcificados en la arteria carótida sobre la radiografía panorámica: difusos, poco visibles, más evidentes en el lado derecho que en el izquierdo. En la evaluación sobre TCHC se hicieron evidentes con mayor claridad, con diferentes tamaños, y sus bordes definidos algunos y otros irregulares. Los ateromas carotideos en nuestro caso en particular, tuvieron apariencias morfológicas similares, variando desde calcificaciones simples a múltiples, estando localizados dentro de los tejidos blandos a nivel del cuello, debajo y posterior al ángulo de la mandíbula. Fue netamente una evaluación radiológica de un paciente en la que no se tomó en cuenta ningún historial clínico, y fue referido al CDI para la evaluación de colocación de implantes. Con relación a este aspecto, Boyle, et al ${ }^{30}$ relataron que el $73 \%$ de todos los pacientes que se refirieron al Departamento de Radiología de Ciencias de la Universidad de Louisville para la evaluación de colocación de implantes se presentaron ateromas de la arteria carótida; todos sobrepasaban la edad de 40 años, y su estado de salud en general se desconocía, pero por su edad, presentaban factores de riesgo vascular, lo cual se asemeja al presente caso porque tampoco se tenía historia clínica al momento de la evaluación, y los ateromas fueron detectados incidentalmente. Es importante resaltar que la paciente en estudio tenia asociado la menopausia y también la edad. Oliveira et al ${ }^{31}$ encontraron en su evaluación de un caso sobre imágenes con TC, ateromas bilaterales bien definidos, que en la radiografía panorámica se evidenciaban, aspecto similar a nuestro caso, por tratarse también de una paciente de sexo femenino de edad avanzada, y presentar ateromas bilaterales. En su revisión encontraron 5 reportes similares al suyo, y concluyeron que la presencia de ateromas bilaterales es frecuente. De este hecho se deriva la importancia del diagnóstico diferencial que se debe dar en una evaluación de imágenes panorámicas y tomográficas para detectar calcificaciones distróficas que se producen en la región maxilofacial.

También es importante conocer la historia clínica general y odontológica del paciente ya que nos puede revelar datos importantes sobre su estado sistémico. Incluso lesiones como la periodontitis, frecuentemente diagnosticada por el odontólogo, también ha sido relacionada con la presencia de placas calcificadas a nivel de carótida. ${ }^{32,33}$ Las características particulares de este caso, con sus imágenes radiográficas y tomográficas bilaterales, deben llevar al clínico a no desestimar el uso de radiografías panorámicas para observar anomalías que no se circunscriben solo a la cavidad bucal y a las estructuras dentales y para dentales, sino también a tener en cuenta hallazgos como los ateromas de carótida, que pueden llegar a tener repercusiones mortales.

Sin embargo, no siempre el Odontólogo tiene el conocimiento para diferenciar la presencia de un ateroma, u otro hallazgo incidental sobre una radiografía panorámica. Por lo tanto, la interconsulta en primer lugar con un radiólogo oral y maxilofacial para descartar o confirmar una sospecha, y en segundo lugar con un cardiólogo, debe ser la conducta de rutina ante la sospecha de que una imagen radiográfica puede constituirse en un ateroma, o en otra entidad de igual o mayor relevancia para la salud de un paciente.

\section{CONCLUSIONES}

1. Un hallazgo incidental de calcificaciones bilaterales en una radiografía panorámica y en la TCHC nos determina que es un poderoso indicador de una situación adversa en el futuro del paciente, ya que a menudo es una manifestación de enfermedades silenciosas que pueden conducir a la muerte.

2. La confirmación de la presencia de un hallazgo incidental sobre una TCHC, da más relevancia a la evaluación tridimensional como herramienta de elección frente a imágenes bidimensionales dudosas.

3. El papel del Radiólogo Oral y Maxilofacial en la consulta clínica diaria, cobra especial relevancia ante cualquier interrogante sobre una imagen radiográfica de difícil clasificación y diagnóstico. 
4. Es importante la interconsulta con cardiología y el manejo interdisciplinario de los pacientes con imágenes sugestivas de calcificaciones intravasculares de carótida, que se descubren en la radiografía panorámica, o en la TCHC, especialmente en aquellos que presentan factores de riesgos asociados a la aterosclerosis, menopausia, diabetes, hipercolesterolemia, obesidad, sedentarismo, consumo de alcohol y entre otros, incluso cuando las imágenes radiográficas no lucen tan evidentes para el odontólogo.

\section{REFERENCIAS BIBLIOGRÁ- FICAS}

1. Friedlander AH, Lande A. Panoramic radiographic identification of carotid arterial plaques. Oral Surg Oral Med Oral Pathol. 1981; 52(1): 102-104.

2. Friedlander AH, Altman L. Carotid artery atheromas in postmenopausal women. Their prevalence on panoramic radiographs and their relationship to atherogenic risk factors. J Am Dent Assoc. 2001; 132(8): 1130-1136.

3. Freymiller EG, Sung EC, Friedlander AH. Detection of radiation-induced cervical atheromas by panoramic radiography. Oral Oncol. 2000; 36(2): 175-179.

4. Arthur H. Friedlander, and Stanley N. Cohen. Panoramic radiographic atheromas portend adverse vascular events. Oral Surg Oral Med Oral Pathol Oral Radiol Endod 2007; 103:830-5.

5. Romano-Sousa CM, Krejci L, Medeiros FM, Graciosa-Filho RG, Martins MF, Guedes VN, Fenyo-Pereira M. Diagnostic agreetment between panoramic radiograph and color doppler images of carotid atheroma. J Appl Oral Sci. 2009; 17(1):45-8.

6. Friedlander, AH, Baker JD. Panoramic radiography: an aid in detecting patients at risk of cerebrovascular accident. J Am Dent Assoc. 1994; 125(12): 15981603.

7. Friedlander AH, Friedlander IK, Yueh R, Littner MR. The prevalence of carotid atheromas seen on panoramic radiographs of patients with obstructive sleep apnea and their relation to risk factors for atherosclerosis. J Oral Maxillofac Surg. 1999; 57(5): 516-522.

8. Friedlander AH, Golub MS. The significance of carotid artery atheromas on panoramic radiographs in the diagnosis of occult metabolic syndrome. Oral Surg Oral Med Oral Pathol Oral Radiol Endod. 2006; 101(1): 95-101.

9. Senosiain-Oroquieta A, Pardo-López B, De Carlos-Villafranca F, Cobo-Plana J. Detección de Placa de Ateroma mediante Radiografías Dentales. RCOE, 2006;11(3):297-303.

10. Lewis DA, Brooks SL. Cartoid artery calcification in a general dental population: a retrospective study of panoramic radiographs. Gen Dent. 1999; 47(1):98103.

11. Manzi FR, Boscolo FN, de Almeida SM, Haiter Neto F. Panoramic radiography as an auxiliary in detecting patients at risk for cerebrovascular accident (CVA): a case report. J Oral Sci. 2003; 45(3):177-80.

12. Cohen SN, Friedlander AH, Jolly DA, Date L. Ca rotid calcification on panoramic radiographs: an important marker for vascular risk. Oral Surg Oral Med Oral Pathol Oral Radiol Endod. 2002; 94 (4):510-4.

13. Tanaka T, Morimoto Y, Ansai T, et al. Can the presence of carotid artery calcification on panoramic radiographs predict the risk of vascular diseases among 80-year-olds? Oral Surg Oral Med Oral Pathol Oral Radiol Endod. 2006; 101:777-783.

14. Carter LC, Tsimidis K, Fabiano J. Carotid calcifications on panoramic radiography identify an asymptomatic male patient at risk for stroke. Oral Surg Oral Med Oral Pathol Oral Radiol Endod. 1998; 85:119122.

15. Mupparapu M. Kim IH. Calcified carotid artery atheroma and stroke: a systematic review. J Am Dent Assoc. 2007 ; $138: 483-492$.

16. Madden RP, Hodges JS, Salmen CW, Rindal DB, Tunio J, MichalowiczBS, et al. Utility of panoramic radiographs in detecting cervical calcified carotid atheroma. Oral Surg Oral Med Oral Pathol Oral Radiol Endod 2007; 103(4): 543-8.

17. Taheri JB, Moshfeghi M. Prevalence of calcified carotid artery on panoramic radiographs in postmenopausal women. J Dent Res Dent Clin Dent Prospect. 2009; 3:46-51.

18. Carter LC. Discrimination between calcified triticeous cartilage and calcified carotid atheroma on panoramic radiography. Oral Surg Oral Med Oral Pathol Oral Radiol Endod. 2000; 90(1):108-10.

19. Kamikawa R S, Pereira M F, Fernandes A, \& Meurer M I, Study of the localization of radiopacities similar to calcified carotid atheroma by means of panoramic 
radiography. Oral Surg. Oral Med. Oral Pathol. Oral Radiol. Endod. 2006;101(3):374-8.20. W h i t e , S. \& Pharoah, M. Radiologia Oral: Principios e interpretación radiográfica. 4a ed. Madrid, Harcourt, 2002. Pp.552-65.

21. Friedlander A H, Manesh F, Wasterlain C G Prevalence of detectable carotid artery calcification on panoramic radiographs of recent stroke victims. Oral Surg. Oral Med. Oral Pathol. 1994; 77(6):669-73.

22. Friedlander A.H. Panoramic radiographs: The differential diagnosis of carotid artery atheromas. Spec Care Dentist. 1995; 15:223-7.

23. Almog DM, Tsimidis K, Moss ME, et al. Evaluation of a training program for detection of carotid artery calcifications on panoramic radiographs. Oral Surg Oral Med Oral Pathol Oral Radiol Endod. 2000; 90:111-7.

24. Almog DM, Padberg FT Jr, Carmel G, Friedlander AH. Previously unappreciated carotid artery stenosis diagnosed by cone beam computerized tomography. J Oral Maxillofac Surg. 2013 Apr; 71(4):702-5. Doi: 10.1016/j.joms.2012.09.021. Epub 2012 Dec 12.

25. Németh Bertalan, Pataky L, Arpád JF, Koppany F, Barabás J. Calcifications in the maxillofacial area. Article in Hungarian. Fogorv Sz. 2015 Sep; 108(3):81-6.

26. Aspestrand F, Kolbenstvedt A. Calcifications of the palatine tonsillary region: CT demonstration. Radiology. 1987; 165:479-480.

27. Khan Z, Wells A, Scarfe W, Farman A. Cone beam
CT isolation of calcifications in the maxillofacial and cervical soft tissues: a retrospective analysis. Int J Computer Assist Radiol Surg.2008; 3(Suppl 1): $\mathrm{S} 221-\mathrm{S} 222$.

28. Magliocca KR, Helman JI. Obstructive sleep apnea: diagnosis, medical management and dental implications. J Am Dent Assoc. 2005; 136:1121-9.

29. Rodríguez-Lozano FJ, Sáez-Yuguero M R, Linares Tovar E, Bermejo Fenoll A. Sleep apnea and mandibular advancement device. Revision of the literature. Med Oral Patol Oral Cir Bucal. 2008; 13:549-54.

30. Boyle, E, M Emerging Trends in Cone Beam Computed Tomography Utilization. [Masters of Science in Oral Biology]. University of Louisville Graduate School, May, 2010.

31. Alves de Oliveira N, Pimenta Amaral T.M, Neves Abdo E, Alves Mesquita R. Bilateral tonsilloliths and calcified carotid atheromas: case report and literatura review. Journal of Cranio Maxillo Facial Surgery. 2013;41:179-182.

32. López FR, Oyarzún MM, Naranjo CC. Asociación entre periodontitis y enfermedad cardiovascular. Rev. Méd Chile. 2000; 128 (11): 1295-6.

33. Janket SJ, Baird AE, Chuang SK, Jones JA. Me ta-analysis of periodontal disease and risk of coronary heart disease and stroke. Oral Surg Oral Med Oral Pathol Oral Radiol Endod 2003; 95 (5):559-69.

Correspondencia: Yris Elisa Chávez Lazo Email: irischavez@yahoo.com 